\title{
Analysis and Design of Networked Control Systems using the Additive Noise Model Methodology
}

\author{
Graham C. Goodwin, Eduardo I. Silva and Daniel E. Quevedo*
}

September 22, 2010

\begin{abstract}
Networked Control has emerged in recent years as a new and exciting area in systems science. The topic has many potential applications in diverse areas ranging from control of microrobots to biological and economic systems. The supporting theory is very rich and combines aspects of control, signal processing, telecommunications and information theory. In this paper, we give a partial overview of recent developments in Networked Control with an emphasis on the additive noise model methodology. We also point to several open problems in this emerging area.
\end{abstract}

Keywords: Networked Control, Performance, Signal-to-noise ratio constraints, Data-Rate Constraints, Delays, Data Dropouts.

\section{Introduction}

Traditionally, control theory has dealt with situations where the communication links between plant and controller can be regarded as transparent (see, e.g., [22,67, 74]). There exist, however, cases where the links in a control system are far from being transparent and may become bottlenecks in the

*G.C. Goodwin and D.E. Quevedo are with the School of Electrical Engineering and Computer Science, University of Newcastle, Callaghan, NSW 2308, Australia. Emails: Graham.Goodwin@newcastle.edu.au, dquevedo@ieee.org. E.I. Silva is with the Departamento de Electrónica, Universidad Técnica Federico Santa María, Casilla 110-V, Valparaíso, Chile. Email: eduardo.silva@usm.cl. A preliminary version of this paper was presented as a plenary address at the Chinese Control and Decision Conference, Yantai, China, July 2008. 
achievable performance. Control systems where this happens are collectively referred to as Networked Control Systems (NCSs).

The study of NCSs has emerged as an active research field during the past years (see, e.g., the special issues $[1,2])$. Key questions within this framework are related to the way in which network artifacts affect the stability and performance of control loops employing non-transparent communication channels. Typical channel artifacts include data-rate limits, random delays and data dropouts. A unifying framework for the treatment of general NCS analysis and design problems does not yet exist. Nevertheless, there has been significant progress in the study of several subproblems. For example, data-rate constraints have been investigated in, e.g., $[43,45,54,70]$. The issue of data dropouts has been studied in, e.g., [36, 55, 57] and random time delays have been considered in, e.g., [35, 46].

A pivotal issue in any closed loop system is that of stability. When focusing on quantization aspects, a key recent result establishes necessary and sufficient conditions on the channel data-rate that allows one to achieve closed loop stability (in an appropriate sense; see, e.g., [45] and the many references therein). These results are expressed in terms of a lower bound on the channel data-rate (that depends on the unstable plant poles only) over which control and coding schemes can be constructed so as to achieve stability. These coding schemes are quite involved and may not be attractive from a practical point of view. This fact has motivated the current authors and colleagues [59, 60] to study simple coding schemes that achieve rates close to the bounds identified above. In [59,60], an important link is established between the average data-rate necessary to achieve stability and signalto-noise ratio requirements for stability. This observation reinforces the relevance of signal-to-noise ratio models in the context of NCS performance analysis (see also [23]), and complements the work reported in $[10,20,52]$, where stabilization over (analog) Gaussian channels is addressed. The first part of this paper reviews these results.

The second part of this paper is devoted to the design of control systems subject to signal-to-noise ratio constraints. For SISO LTI plant models, we show how one can design coding schemes that, for a given initial non-networked controller design, allow one to minimize the impact of signal-to-noise ratio constraints on overall closed loop performance. These results emphasize the essential role that the available degrees of freedom play in NCSs (see $[23,63,65]$ and also $[4,11,12,38,41])$.

The issue of performance is particularly interesting in the context of networked control architectures for MIMO plant models. Practical control systems for MIMO plant models often use structurally constrained controllers such as diagonal or triangular ones (see, e.g., [53,67]). The reasons for this choice 
are manifold and include ease of design, simplified tuning, and implementation related aspects such as cabling or geographic plant distribution. It is well known that restricting the controller architecture generally limits the achievable performance (see, e.g., $[24,34,64]$ ). Within this context, networks can play significative roles. Indeed, it is easy to envisage decentralized control architectures that, when enriched with additional data paths implemented via (non-transparent) communication links, may provide enhanced performance. This may (partially) overcome the limitations that arise as a consequence of the controller structure constraint (see, e.g., $[30,32,40,51,71]$ ). These ideas are illustrated in the present paper by examining the control of MIMO LTI systems for which a decentralized controller has been successfully designed. Unsurprisingly, for high-quality additional channels it turns out that a networked MIMO architecture outperforms the decentralized one. However, an interesting finding is that, in some situations, the networked architecture will perform better than the decentralized one only if the channels used to implement the scheme are extremely reliable [61].

Finally, we will briefly mention other networked control problems which lie outside the signal-tonoise ratio paradigm. In particular, we refer to the problems associated with data dropouts and delays (see, e.g., [5, 13, 27, 29, 36, 37, 49,55,68]). Provided one can send additional information at each sample time, then this can be used to provide robustness against data-loss or delay [50].

The remainder of this paper is organized as follows: Section 2 motivates some of the problems to be addressed in the paper. Section 3 discusses the problem of stabilization of NCSs subject to data rate constraints. Section 4 discusses the design of coding systems that optimize NCS performance. Section 5 studies some aspects in MIMO networked control. Section 6 studies control over unreliable networks. Section 7 draws conclusions.

\section{A Simple Motivational Example}

Before delving into the problem of NCSs, we will first motivate the key claim that networked control raises issues that go beyond traditional control theory. The analysis in this section will contain a number of heuristic steps. In the remainder of the paper, we will show how similar questions can be addressed in a rigorous fashion.

We consider a simple SISO feedback loop as depicted in Figure 1. In that figure, we have placed a (communication) channel in the down-link between the controller $C(z)$ and the plant $G(z)$.

Figure 1 immediately gives rise to an important question, namely how should we model the channel. 


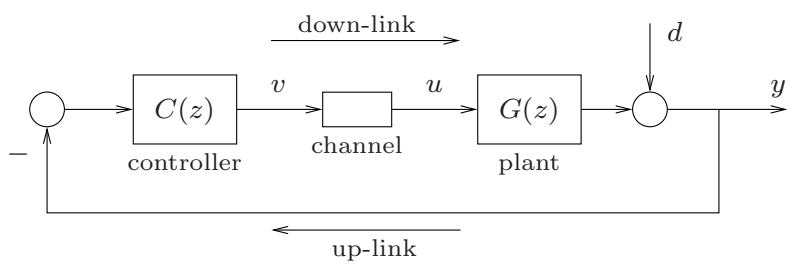

Figure 1: A simple networked control system.

There are many options, but for the purpose of our current motivational study, we will simply model the channel as a bit-rate limited system that sends $b$ bits per sample. We assume that no other sources of non-ideal behavior exist.

We will be particularly interested in how the channel affects closed loop performance. Thus, we will focus on the errors introduced by the channel. We will adopt a very simple model for quantization errors, namely that they are independent, white and uniformly distributed on $\left(-\frac{\Delta}{2}, \frac{\Delta}{2}\right)$, where $\Delta$ is the quantizer step size (see, e.g., $[7,31,56]$ ). A simple calculation shows that the variance of the quantization errors, say $\sigma_{q}^{2}$, is then given by

$$
\sigma_{q}^{2}=\frac{\Delta^{2}}{12}
$$

Next we need to determine $\Delta$. If we fix the quantizer step size and the number of bits, then we also fix the (one-sided) range of the quantizer to be $\left(2^{b}-1\right) \frac{\Delta}{2}$. Heuristically, this (one-sided) range needs to be sufficiently large to be able to deal with the incoming signal $v$. We will adopt the, so-called, $4 \sigma_{v}$ rule $^{1}$ which sets the (one-sided) range of the quantizer to be equal to four times the standard deviation of the signal being quantized, namely $v$, so as to make quantizer overload a low probability event. This leads to the following relationship linking $\Delta, b$ and $\sigma_{v}^{2}$ :

$$
\frac{\left(2^{b}-1\right) \Delta}{2}=4 \sigma_{v}
$$

Using (1) and (2) we obtain

$$
\frac{\sigma_{v}^{2}}{\sigma_{q}^{2}}=\frac{3}{16}\left(2^{b}-1\right)^{2}=\gamma,
$$

where $\gamma$ is a constant. Thus, we see that our assumptions have led to the conclusion that the (nontransparent) channel effectively introduces an additional white noise source whose variance is pro-

\footnotetext{
${ }^{1}$ Commonly used in the signal processing literature [31].
} 


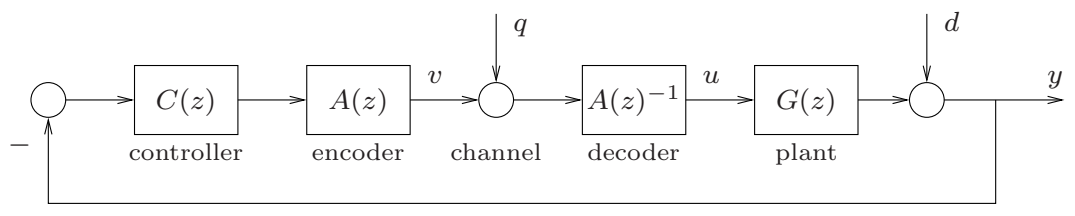

Figure 2: Networked control system with linear coding for additive noise channel.

portional to the variance of the channel input. In other words, our assumptions lead to a fixed signal-to-noise ratio additive noise channel model.

With the benefit of hindsight, we realize that it is desirable to pre-process the signal before sending it through the channel, i.e., before quantizing it. Here, we will adopt the simplest possible pre-processor, namely a bi-proper filter having transfer function $A(z)$. Also, we do not want the filter to affect the nominal feedback loop, so we will also include $A(z)^{-1}$ at the output of the channel. Putting the above elements together leads to the modified feedback loop shown in Figure $2 .^{2}$

It is obvious from Figure 2 that the filter $A(z)$ plays no role in the nominal performance, i.e., when $q=0$. However, we will see below that $A(z)$ does affect the networked performance when $q \neq 0$. Specifically, we will be interested in the (stationary) variance of $y$ due to $q$, which we will denote by $\sigma_{e}^{2}$. A simple calculation, using (3), gives

$$
\sigma_{e}^{2}=\frac{\sigma_{q}^{2}}{2 \pi} \int_{-\pi}^{\pi}\left|\frac{A\left(e^{j \omega}\right)^{-1} G\left(e^{j \omega}\right)}{1+G\left(e^{j \omega}\right) C\left(e^{j \omega}\right)}\right|^{2} d \omega=\frac{\sigma_{v}^{2}}{2 \pi \gamma} \int_{-\pi}^{\pi}\left|\frac{A\left(e^{j \omega}\right)^{-1} G\left(e^{j \omega}\right)}{1+G\left(e^{j \omega}\right) C\left(e^{j \omega}\right)}\right|^{2} d \omega .
$$

Finally, we need to evaluate $\sigma_{v}^{2}$. Here we will make the assumption that $\sigma_{v}^{2}$ is mainly affected by the disturbance $d$, which we assume is a wide sense stationary (wss) process. ${ }^{3}$ Hence we have

$$
\sigma_{v}^{2}=\frac{1}{2 \pi} \int_{-\pi}^{\pi}\left|\frac{C\left(e^{j \omega}\right) A\left(e^{j \omega}\right) \Omega_{d}\left(e^{j \omega}\right)}{1+G\left(e^{j \omega}\right) C\left(e^{j \omega}\right)}\right|^{2} d \omega
$$

where $\Omega_{d}\left(e^{j \omega}\right)$ is a spectral factor of $d[3]$. Substituting (5) into (4) yields

$$
\sigma_{e}^{2}=\frac{1}{\gamma}\left(\frac{1}{2 \pi} \int_{-\pi}^{\pi}\left|\frac{A\left(e^{j \omega}\right)^{-1} G\left(e^{j \omega}\right)}{1+G\left(e^{j \omega}\right) C\left(e^{j \omega}\right)}\right|^{2} d \omega\right)\left(\frac{1}{2 \pi} \int_{-\pi}^{\pi}\left|\frac{C\left(e^{j \omega}\right) A\left(e^{j \omega}\right) \Omega_{d}\left(e^{j \omega}\right)}{1+G\left(e^{j \omega}\right) C\left(e^{j \omega}\right)}\right|^{2} d \omega\right) .
$$

An elementary application of the Cauchy-Schwartz inequality now shows that $\sigma_{e}^{2}$ is minimized if we

\footnotetext{
${ }^{2}$ In Figure 2 we use the term encoder and decoder to refer to $A(z)$ and $A(z)^{-1}$, respectively. This is consistent with standard signal processing nomenclature (see, e.g., [16] and the references therein).

${ }^{3}$ Unless otherwise stated, all signals in this paper are assumed wss processes.
} 


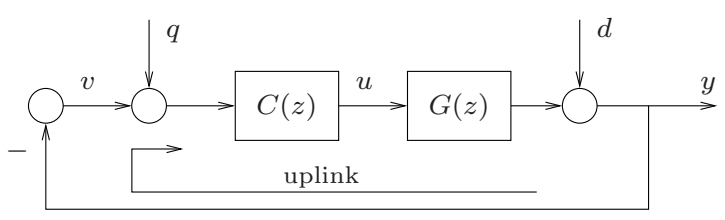

Figure 3: Networked control system of Figure 2 with a particular optimal coding scheme (see text for details).

can choose $A(z)$ to satisfy, for every $\omega \in[-\pi, \pi]$,

$$
\left|A\left(e^{j \omega}\right)^{-1} G\left(e^{j \omega}\right)\right|^{2}=k_{1}\left|C\left(e^{j \omega}\right) A\left(e^{j \omega}\right) \Omega_{d}\left(e^{j \omega}\right)\right|^{2} \Leftrightarrow\left|A\left(e^{j \omega}\right)\right|^{4}=\frac{1}{k_{1}}\left|\frac{G\left(e^{j \omega}\right)}{C\left(e^{j \omega}\right) \Omega_{d}\left(e^{j \omega}\right)}\right|^{2},
$$

where $k_{1}$ is a constant (see also [23]).

A very interesting special case of practical importance arises when $d$ is step-like and we model it as a random walk, i.e.,

$$
\Omega_{d}\left(e^{j \omega}\right)=\frac{1}{e^{j \omega}-1}
$$

Also, if the plant is stable and minimum phase, then we can use a controller $C(z)$ such that

$$
G(z) C(z)=\frac{c z^{-n}}{z-1}
$$

where $n$ is a suitable positive integer and $c$ is a constant. These choices for $C(z)$ and $\Omega_{d}\left(e^{j \omega}\right)$ lead to

$$
A(z)=k_{2} C(z)^{-1}
$$

where $k_{2}$ is an arbitrary constant. This result implies that, for the considered architecture, controller and disturbance description, the optimal pre-processor of the signal $v$ is the inverse of the controller. If we can change the architecture, then it is sufficient to put the channel in the up-link, since Figure 2 reduces to Figure $3 .^{4}$

The reader will see that many of the ideas presented above will reappear in the sequel. For example, examining Figure 4 we see that the communication link has been placed in the up-link and that preand post-processing has been added (see $\mathcal{E}$ and $\mathcal{D}$ in Figure 4 ).

Whilst the above motivational study is very simple, it does lead to many important questions:

\footnotetext{
${ }^{4}$ Actually, it was a practical experiment that originally led the authors to this remarkably simple observation [47].
} 
- Is it ever justifiable to model quantization errors as white noise?

- How can one treat the fact (ignored above) that quantization errors also affect the channel (quantizer) input $v$ ?

- Are there better coder-decoder pairs than simple filters?

- Can one have a rigorous alternative to the $a d-h o c 4 \sigma$-rule?

- Should one redesign the controller $C(z)$ or, perhaps, carry out joint design of the controller and coder-decoder pair?

- Are there situations where the presence of a communication channel can destabilize a nominally stable loop?

- What if packet loss occurs (i.e., not all transmitted data is received)?

We will rigorously address many of these issues below, beginning with the key question of closed loop stability.

\section{$3 \quad$ Stability with Data-rate Limits}

An overarching issue in any closed loop system is the potential for instability. For this reason, obtaining necessary and sufficient conditions which guarantee closed loop stability has remained a central issue in control theory (see, e.g., [22]). NCSs give rise to new challenges with respect to stability. This section will give some insights into NCS stability questions.

Consider the networked control situation shown in Figure 4. In that figure, $G(z)$ is a SISO LTI plant, $C(z)$ is an LTI controller, $r$ is a reference signal and $d$ is a disturbance. Unlike standard nonnetworked control systems (see, e.g., $[22,67,74]$ ) the feedback path in the control system in Figure 4 comprises a non-transparent channel and a coding scheme. The coding scheme is a novel aspect of networked control that has no equivalent in traditional control theory. The coding scheme has two parts: the encoder and the decoder. The encoder is in charge of appropriately processing the signal to be sent over the channel so as to compensate, if possible, channel characteristics and/or to translate the measurements into symbols that the channel can understand. (This is the case of, e.g., digital channels 




Figure 4: Basic networked control system (including coding scheme).

where the symbols are binary words.) On the other hand, the decoder is in charge of translating back the channel symbols into the standard signal domain.

There exist many channel models (see, e.g., [14,69]). To highlight the ideas, in the present section we will consider error-free and delay-free digital channels, i.e., channels that can transmit a countable set of symbols (i.e., that have a countable alphabet) without errors or delays. Of course, in practice, channels always have restricted bandwidth and the channel alphabet is, thus, finite, rather than countable (see also $[15,45,48])$.

\subsection{Key results}

For the setting described above, a recent result states that it is possible to find coders, decoders and controllers such that the resulting system is mean square stable (MSS) if and only if the asymptotic time-average length of the channel symbols in bits per sample, say $\bar{R}_{T}$, satisfies [43]

$$
\bar{R}_{T}>\sum_{i=1}^{n_{p}} \log _{2}\left|p_{i}\right|,
$$

where $\left\{p_{i}\right\}_{i=1, \cdots, n_{p}}$ denotes the set of unstable plant poles.

The above result is valid for every coder, decoder and controller in the class of (possibly timevarying and nonlinear) causal systems. Therefore, (11) establishes a fundamental separation between what is achievable in NCSs over digital channels and what is not, when the problem of interest is MSS. We note that bounds similar to (11) arise as solutions to quite a few different problems (e.g., observability, deterministic stability, etc.) and under different assumptions on the channels and coding schemes (see, e.g., $[19,28,42,43,69,70])$. Consequently, the quantity on the right hand side of (11) can be seen to be a fundamental measure of the difficulty of manipulating a system, as explored in [44,54].

Proving that the rate at which a stable control system is transmitting data must satisfy (11) (i.e., 




$\mathcal{D}$

$\varepsilon$

Figure 5: Proposed coding scheme for stabilization.

necessity) is fairly simple and employs standard tools (see also [17,21,39]), whereas constructing an actual coding scheme that achieves stability at any rate above the absolute limit (i.e., the proof of sufficiency) is much more involved (see $[43,70]$ ). These observations motivate the remainder of this section.

\subsection{Some insights into the problem of stabilization with data-rate con- straints}

In this section we provide insights into the limitations that finite data-rates impose. To fix ideas, we will consider the coding scheme depicted in Figure 5, where $F(z)$ is a LTI filter, and $\overline{\mathcal{E}}$ and $\overline{\mathcal{D}}$ are abstract systems that translate the discrete time signal $v$ into channel symbols $s$, and channel symbols into the discrete time signal $w$, respectively. ${ }^{5}$ Our presentation will start at a heuristic level and will then move towards a more formal approach based on information theoretic considerations.

\subsubsection{Additive noise model for quantization}

Since the channel is digital, $\overline{\mathcal{E}}$ must quantize $v$ prior to transmission. We will focus on the simplest situation where $\overline{\mathcal{D}}$ is the identity and $\overline{\mathcal{E}}$ is a finite uniform quantizer (see, e.g., [25,31]), i.e., ${ }^{6}$

$$
s(k)=\mathcal{Q}(v(k)) \triangleq \operatorname{sat}_{V}\left(\Delta\left\lfloor\frac{v(k)}{\Delta}\right\rfloor\right) .
$$

In (12), $V>0$ is the quantizer dynamic range, $\Delta \triangleq 2 V(L-1)^{-1}$ is the quantization step and $L$ is the number of quantization levels. We say the the quantizer is overloaded if and only if $|v(k)|>V$ for some $k$. (If in (12) $\operatorname{sat}_{V}$ is removed and $\Delta$ is fixed at any arbitrary positive value, then $\mathcal{Q}$ becomes an infinite uniform quantizer with a countable output set.)

\footnotetext{
${ }^{5}$ Figure 5 represents a generalization of the simple filter plus quantizer architecture used in Section 2.

${ }^{6}$ sat $_{V}(x) \triangleq x$ if $|x| \leq V$ and $\operatorname{sat}_{V}(x) \triangleq \frac{x}{|x|} V$ if $|x|>V ;\lfloor x\rfloor$ denotes the integer part of $x$.
} 
Quantization is a non-linear operation and hence, the exact analysis of quantized systems is difficult (see, e.g., $[15,26,48]$ ). Thus, and as already mentioned in Section 2, it has become standard, particularly in the signal processing literature (see, e.g., $[7,31,56]$ ), to (approximately) describe the quantization errors, defined via

$$
q \triangleq w-v
$$

by an additive i.i.d. noise source, uniformly distributed on the interval $\left(-\frac{\Delta}{2}, \frac{\Delta}{2}\right)$ and independent ${ }^{7}$ of the input to the quantizer $v$. This model is valid only if $\Delta$ is "small enough", the quantizer does not overload and $v$ has a smooth probability density function (see, e.g., [6]).

In order to avoid quantizer overload, it is usual in practice to choose a quantizer dynamic range (i.e., one-sided range) such that the probability of overload is negligible [31]. Indeed, if $v$ is wss and $\beta$ is any positive real value, then one can always find a finite $\alpha$ such that ${ }^{8} V=\alpha \sigma_{v}$ guarantees that the probability of overload is less than $\beta ; \alpha$ is called the quantizer loading factor. ${ }^{9}$ With such a choice for the loading factor, it is immediate to see that, provided $q$ is as described above, then

$$
\gamma \triangleq \frac{\sigma_{v}^{2}}{\sigma_{q}^{2}}=\frac{3}{\alpha^{2}}(L-1)^{2},
$$

where $\gamma$ is the quantizer signal-to-noise ratio. Thus, at least at an heuristic level, bit-rate limits translate into signal-to-noise ratio constraints.

It is important to note that the previous model for quantization can actually be rendered exact by a simple randomization procedure. Indeed, it suffices to consider a dithered uniform quantizer. In such a quantizer, one adds a specially designed random process, the so-called dither signal, to the signal being quantized and then subtracts it at the receiving end. More precisely, if $d_{h}$ denotes the dither signal, then we have

$$
\begin{aligned}
& s(k)=\operatorname{sat}_{V}\left(\Delta\left\lfloor\frac{v(k)+d_{h}(k)}{\Delta}\right\rfloor\right), \\
& w(k)=s(k)-d_{h}(k),
\end{aligned}
$$

where the $d_{h}$ is chosen i.i.d., uniformly distributed on $\left(-\frac{\Delta}{2}, \frac{\Delta}{2}\right)$ and such that $d_{h}(k)$ is independent of $v(k)$. In this situation, and provided no overload occurs, $q$ in (13) becomes distributed as $-d_{h}$ (see,

\footnotetext{
${ }^{7}$ Sometimes $q$ is assumed only to be uncorrelated with $v$.

${ }^{8} \sigma_{v}$ denotes the standard deviation of $v$.

${ }^{9}$ For example, if $v(k)$ is Gaussian, then $\alpha=4$ gives an overload probability of $6.33 \cdot 10^{-5}$.
} 
e.g., $[25,72,73])$. In other words, $q$ has the properties in the simplified model described above. Again, in order to render quantizer overload a low probability event, one needs to consider an appropriate quantizer loading factor and a signal-to-noise ratio constraint arises. In practice, implementation of dithered quantizers is not trivial since it requires the availability of the dither signal $d_{h}$ at both the sending and receiving ends. Usually the dither is generated using pseudo-random number generators that are initialized with the same seeds.

The above quantization model does not in general hold in the case of non-dithered quantizers. Nevertheless, even in that case, if one employs a uniform quantizer with as small as, say, 4 levels, it turns out that the predictions made using the simple additive quantization model described above are surprisingly accurate (see simulation studies in $[16,23,56,63]$ ).

\subsubsection{Limits imposed by signal-to-noise ratio constraints}

Consider the setting in Figure 5 and assume that $\overline{\mathcal{E}}$ and $\overline{\mathcal{D}}$ are such that $q$ obeys the additive model for quantization described in Section 3.2.1. We note that, since $q$ depends on the way in which $\bar{\varepsilon}$ and $\overline{\mathcal{D}}$ are designed, the variance of $q$, say $\sigma_{q}^{2}$, becomes a decision variable. ${ }^{10}$ Within this setting, a basic question arises, namely finding the conditions on the signal-to-noise ratio $\gamma$ that guarantee MSS.

It is possible to show via standard control theoretic arguments [60] that, for any strictly proper plant model $G(z)$ and any wss processes $r$ and $d$, there exist $C(z), F(z)$ and a finite $\sigma_{q}^{2}$ such that the resulting NCS is MSS if and only if

$$
\gamma>\gamma_{\mathrm{inf}} \triangleq\left(\prod_{i=1}^{n_{p}}\left|p_{i}\right|^{2}\right)-1
$$

where $\left\{p_{1}, \cdots, p_{n_{p}}\right\}$ is the set of unstable plant poles. From this result we conclude that, as was the case for (11), the degree of instability of the plant plays a key role in the interplay between stability and communications constraints (as expressed by signal-to-noise ratio constraints in our current framework). It is interesting to note that if one adds one and takes logarithms in (15), then one recovers the right hand side in (11). We have shown in [60] that this connection is not a mere coincidence (see also [59]).

It is easy to see from (15) and (14) that, if the additive model for quantization holds, then MSS is

\footnotetext{
${ }^{10}$ For the additive model described previously, we have $\sigma_{q}^{2}=\frac{\Delta^{2}}{12}$.
} 
equivalent to

$$
b>b_{\mathrm{inf}} \triangleq \log _{2}\left(1+\sqrt{\frac{\alpha^{2}}{3}\left(\left(\prod_{i=1}^{n_{p}}\left|p_{i}\right|^{2}\right)-1\right)},\right.
$$

where $b \triangleq \log _{2} L$ is the number of bits in the quantizer. In other words, provided no overload occurs and the noise model for quantization holds, (16) gives a bound on the instantaneous data-rate over the channel that guarantees MSS. ${ }^{11}$ Clearly, this bound on $b$ is optimistic. Indeed, it is only exact if one employs a dithered uniform quantizer and there is no overload. Note that, if $r, d$ or the initial plant state have unbounded support, then it is impossible to guarantee that the input to the quantizer is deterministically bounded. Even if $r, d$ and the plant initial state have bounded support, and thus $v$ is bounded, the previous analysis usually requires a large value for the loading factor $\alpha$. Equation (16) then provides only a conservative bound on $b_{\text {inf }}$.

In practice it is often sufficient to assume that $v$ is such that a sensibly small value for $\alpha$ gives negligible overload probability (a typical value is $\alpha=4$; see [31] and also Sections 4 and 5).

\subsubsection{Average data-rates}

In the previous section we dealt with instantaneous data-rates. There are at least two reasons that can be advanced for considering alternative settings. First, if the external signals have unbounded support, then it is impossible to give any guarantees using the above reasoning, unless one employs quantizers with infinitely many levels so as to avoid overload (thus, incurring infinite instantaneous data-rates). Second, if the external signals are bounded, then the quantizer loading factor $\alpha$ may need to be quite large in order to accommodate $v$ without overload. This implies that, even in those cases, one needs to use a quantizer with either a large number of bits (which increases the instantaneous rate), or a large quantization step (which implies poor performance). We thus conclude that, at least from a theoretical point of view, it is interesting to study average data-rates. ${ }^{12}$ (Of course, guaranteeing that average data-rates are bounded does not ensure that instantaneous rates will be bounded.)

\footnotetext{
${ }^{11}$ Clearly, $b_{\text {inf }}$ is in general greater than the right hand side in (11).

${ }^{12}$ Alternatively, one could also examine time varying (i.e., adaptive) quantizers that are scaled on-line according to the statistics of the input signals. This topic is interesting, but is beyond the scope of this paper (see references in [45]).
} 


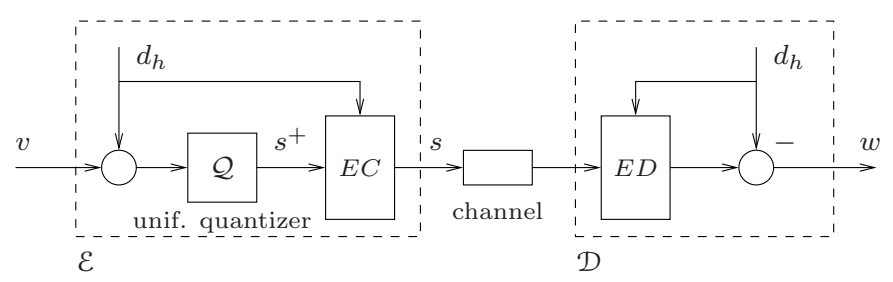

Figure 6: Entropy coded dithered quantizer.

We will measure average data-rates via

$$
\bar{R} \triangleq \lim _{k \rightarrow \infty} \frac{1}{k} \sum_{i=0}^{k-1} R(i),
$$

where $R(i)$ is the expected length of the symbol sent at time instant $i$ over the channel, i.e., of $s(i)$ (see [59] and also $[14,58]$ ). In the sequel, we will reserve the term average data-rate to refer to $\bar{R}$ in (17). Note that $\bar{R}$ in (17) is not equal to $\bar{R}_{T}$ in (11). However, under certain regularity conditions ${ }^{13}$ $\mathcal{E}\left\{\bar{R}_{T}\right\}=\bar{R}$ (as revealed by the corresponding definitions).

A popular way to deal with average data rate constraints is to use an entropy coded dithered quantizer (ECDQ; see, e.g., [72]). Figure 6 shows the architecture of an ECDQ and its relationship to $\overline{\mathcal{E}}$ and $\overline{\mathcal{D}}$ in Figure 5. An ECDQ uses a dithered infinite uniform quantizer with step $\Delta$, as described earlier, but instead of sending the quantizer output directly over the channel, it entropy-codes it prior to transmission. The basic idea behind the entropy-coder EC in Figure 6 is to exploit the fact that some of the quantizer output values are more likely than others. Thus, one can assign short channel symbols (i.e., few bits) to very frequent quantizer output values and long symbols (i.e., many bits) to infrequent ones [14]. Correspondingly, the task of the entropy-decoder ED is to convert the channel symbols back into the exact quantizer output at the receiving end.

A key property of ECDQs is that the average data-rate at which data is transmitted can be related to the signal-to-noise ratio

$$
\gamma=\frac{\sigma_{v}^{2}}{\sigma_{q}^{2}}
$$

where $\sigma_{q}^{2}$ is the variance of the equivalent additive noise in the dithered quantizer, and $\sigma_{v}^{2}$ is the variance of the input to the ECDQ, namely $v$. Indeed, it is possible to show that, if the dither signal

\footnotetext{
${ }^{13} \mathcal{E}\{\cdot\}$ denotes the expectation operator.
} 
is as in Section 3.2 and if it is also independent of $r, d$ and the filter initial states, then the coding scheme proposed in Figure 5, when $\overline{\mathcal{E}}$ and $\overline{\mathcal{D}}$ form an ECDQ, is such that the average data-rate is upper bounded by [60]

$$
\bar{R} \leq \frac{1}{2} \ln (1+\gamma)+\frac{1}{2} \log _{2}\left(\frac{2 \pi e}{12}\right)+1 .
$$

Thus, we see that signal-to-noise ratio considerations give an upper bound on the average data-rate. In other words, there exists a strong link between signal-to-noise ratio constraints and average data-rate constraints. This goes beyond the heuristic discussion in Section 3.2.1, and serves as a basis for a simple but rigorous treatment of average data-rate constraints in networked control.

In particular, one can use (11), (15), (19), and the relationship between $\bar{R}$ and $\bar{R}_{T}$ mentioned above, to show that it is possible to choose the filter $F(z)$ in the scheme of Figure 5 so that MSS is guaranteed and the average data-rate $\bar{R}$ satisfies [60] (see also related results in [59])

$$
\sum_{i=1}^{n_{p}} \log _{2}\left|p_{i}\right|<\bar{R} \leq \sum_{i=1}^{n_{p}} \log _{2}\left|p_{i}\right|+\frac{1}{2} \log _{2}\left(\frac{2 \pi e}{12}\right)+1+f(\Delta),
$$

where $f$ is a positive function that goes to zero as the quantization step $\Delta$ goes to infinity. Therefore, the use of an ECDQ with a sufficiently large (but finite) quantization step $\Delta$, allows one to achieve MSS at an average rate that can be made arbitrarily close to the absolute minimum rate in (11) plus $\frac{1}{2} \log _{2}\left(\frac{2 \pi e}{12}\right)+1$ bits per sample (i.e., $\approx 1.25$ bits per sample). This additional rate is composed of two terms: the first one is due to the divergence of the distribution of quantization noise from Gaussianity, and the second one originates from the inefficiency of the loss-less coding scheme employed to generate the channel symbols (i.e., the inefficiency of EC; see [14]).

\section{Performance}

Next we turn to the question of performance. Motivated by the analysis presented in the previous section, we will utilize an independent additive noise channel model with a signal-to-noise ratio constraint. This hypothesis covers many situations:

- Architectures where the actual physical channel is additive and with a signal-to-noise ratio constraint.

- Situations where the channel is digital, has a finite alphabet and one assumes that quantization noise is white, independent, and the quantizer is properly scaled (recall Section 3.2.1). 




Figure 7: Linear model for the NCS in Section 4.

- Cases where the channel is digital, has a countable alphabet, and one employs an ECDQ (see Section 3.2.3).

- Situations where the channel is analog, but prone to i.i.d. data dropouts (i.e., analog erasure channel; see also $[18,55,57])$. Indeed, it has been shown in [36,62] (see also Chapter 7 in [59]) that, for such data dropout profiles, analog erasure channels are equivalent, up to second order statistics, to an additive white noise channel with a fixed signal-to-noise ratio.

\subsection{Setup and Analysis}

We will focus on the NCS depicted in Figure 7, which is an extension of the setup studied in Section 2 by the addition of feedback around the channel (quantizer). In that figure, the controller $C(z)$ is any given admissible controller for $G(z) .{ }^{14}$ We will show how to design the coding system filters $F(z)$ and $A(z)$ so as optimize overall closed loop performance. (We refer the reader to [66] for controller design procedures.)

It is easy to see from Figure 7 that the variance of the tracking error, i.e.,

$$
e \triangleq r-y
$$

is given by ${ }^{15}$

$$
\sigma_{e}^{2}=\left\|T_{d r e}(z) \Omega_{d r}(z)\right\|_{2}^{2}+J(A(z), F(z)),
$$

\footnotetext{
${ }^{14}$ i.e., an internally stabilizing controller that defines a well posed control loop (see, e.g., [74]).

${ }^{15}$ We define $\|X(z)\|_{2}^{2} \triangleq \operatorname{trace}\left\{\frac{1}{2 \pi} \int_{-\pi}^{\pi} X\left(e^{j \omega}\right) X\left(e^{j \omega}\right)^{H} d \omega\right\}$.
} 
where

$$
J(A(z), F(z)) \triangleq \frac{\left\|A(z) T_{d r y}(z) \Omega_{d r}(z)\right\|_{2}^{2}\left\|T(z) A(z)^{-1}(1-F(z))\right\|_{2}^{2}}{\gamma-\|T(z)+S(z) F(z)\|_{2}^{2}},
$$

and where $T_{d r e}(z)$ and $T_{d r y}(z)$ denote the transfer functions from $[d r]^{T}$ to $e$ and $y$, respectively, $\Omega_{d r}(z)$ denotes a spectral factor of $[d r]^{T}, \gamma$ is the channel signal-to-noise ratio and

$$
S(z)=\frac{1}{1+G(z) C(z)}, \quad T(z) \triangleq 1-S(z) .
$$

A straightforward calculation shows that $T_{d r e}(z)$ depends on $C(z)$ only, which is fixed in our setting. Thus, to optimize performance we only need to minimize $J(A(z), F(z))$.

A simple calculation shows that, provided $C(z)$ is an admissible controller for $G(z)$, the model in Figure 7 is MSS and well-posed if and only if $F(z)$ is stable, strictly proper, $A(z)$ is stable, minimum phase (MP), biproper, and the signal to noise ratio satisfies

$$
\gamma>\|T(z)+S(z) F(z)\|_{2}^{2}
$$

This implies that, for any given $\gamma$ and even if $C(z)$ is a stabilizing controller for $G(z)$, it is possible to choose a filter $F(z)$ such that the resulting NCS is not MSS. This highlights the interplay between communication aspects (namely, $\gamma$ ) and coder-controller design issues (namely, the choice of $F(z$ ); see also Section 3.2.2).

It is illustrative to note that, if $\gamma \rightarrow\|T(z)+S(z) F(z)\|_{2}^{2}$, then $J(A(z), F(z)) \rightarrow \infty$ (unless all exogenous signals have zero spectral density, in which case $J(A(z) F(z)) \equiv 0)$. Also, if $\gamma \rightarrow \infty$, then $J(A(z), F(z)) \rightarrow 0$ and we recover the non-networked performance achieved with controller $C(z)$ and a transparent communication link.

\subsection{Coding system design}

Based on (22) and noting that $T_{d r w}(z) \Omega_{d r}(z)$ is fixed, we next study the problem of finding ${ }^{16}$

$$
J_{\text {opt }} \triangleq \inf _{\substack{A(z) \in \mathcal{U}_{\infty} \\ F(z) \in \mathcal{R} \mathcal{H}_{2} \\\|T(z)+S(z) F(z)\|_{2}^{2}<\gamma}} J(A(z), F(z))
$$

\footnotetext{
${ }^{16} \mathcal{R} \mathcal{H}_{2}$ denotes the set of all stable and strictly proper real rational transfer functions, and $\mathcal{U}_{\infty}$ denotes the set of all stable, MP and biproper transfer functions.
} 
and filters $A(z)$ and $F(z)$ that achieve $J_{o p t}$ (or approximate $J_{o p t}$ arbitrarily well). This is an extension of the simple optimization problem studied in Section 2. The exact solution of this problem is not straightforward, so we describe a simple iterative solution (see also related results in [16]).

If $F(z)$ is a given strictly proper and stable filter satisfying (23) (as required for MSS), then the reasoning used in Section 2 implies that the optimal filter $A(z)$, say $A_{o p t}^{F(z)}(z)$, satisfies [23,65]

$$
\left|A_{\text {opt }}^{F(z)}\left(e^{j \omega}\right)\right|^{4}=\beta \frac{\left|T\left(e^{j \omega}\right)\left(1-F\left(e^{j \omega}\right)\right)\right|^{2}}{T_{d r y}\left(e^{j \omega}\right) \Omega_{d} r\left(e^{j \omega}\right) \Omega_{d} r\left(e^{j \omega}\right)^{H} T_{d r y}\left(e^{j \omega}\right)^{H}},
$$

where $\beta$ is any arbitrary positive real. We note that the characterization of $A_{o p t}^{F(z)}(z)$ given above is explicit but is in general not satisfied by any stable, biproper and MP rational transfer function. (Indeed, the 4th root of the right hand side in (24) is usually irrational.) Nevertheless, since the condition (24) applies only to the magnitude of $A(z)$, it is always possible to find a filter with the desired characteristics that achieves a performance that is as close as desired to the optimal performance. In practice, it is usually sufficient to consider reasonably low order filters to approximate $A_{\text {opt }}^{F(z)}(z)$ (see also [23]).

Next, let us assume that $A(z)$ is any given stable, MP and biproper transfer function. For any such $A(z)$, it is possible to show that $[65]$

$$
F_{o p t}^{A(z)}(z)=F_{\epsilon^{*}}(z)
$$

where $F_{\epsilon}(z), \epsilon \in[0,1]$, is defined via

$$
\begin{gathered}
F_{\epsilon}(z) \triangleq \arg \inf _{F(z) \in \mathcal{R H}_{2}} \epsilon J_{1}(F(z))+(1-\epsilon) J_{2}(F(z)), \\
J_{1}(F(z)) \triangleq\left\|T(z) A(z)^{-1}(1-F(z))\right\|_{2}^{2}, \quad J_{2}(F(z)) \triangleq\|T(z)+S(z) F(z)\|_{2}^{2},
\end{gathered}
$$

and

$$
\epsilon^{*} \triangleq \arg \min _{\epsilon \in(0, \hat{\epsilon})} \frac{J_{1}\left(F_{\epsilon}(z)\right)}{\gamma-J_{2}\left(F_{\epsilon}(z)\right)} .
$$

In (26), $\hat{\epsilon}$ is defined as follows: If there does not exist an $\epsilon \in(0,1]$ such that $J_{2}\left(F_{\epsilon_{\gamma}}(z)\right)=\gamma$, then $\hat{\epsilon}=1$. Otherwise, $\hat{\epsilon}=\epsilon_{\gamma}$, where $\epsilon_{\gamma}$ is the unique real in $(0,1]$ such that $J_{2}\left(F_{\epsilon_{\gamma}}(z)\right)=\gamma$.

We note that the problem of finding $F_{\epsilon}(z)$ is a standard convex problem that can be tackled using well-known tools (see, e.g., $[8,9]$ ). On the other hand, calculating $\epsilon^{*}$ amounts to carrying out a (scalar) line search. This is also a simple problem to solve. 
By utilizing the previous results, it is immediate to design an iterative procedure that allows one to construct approximations to the optimal $(A(z), F(z))$ pair: In a first step, one fixes $A(z)$ (or $F(z)$ ); trivial choices are $F(z)=0$ and $A(z)=1$. Then, one uses (25) to calculate the optimal $F(z)$ for the initial choice of $A(z)$ (or $(24)$ to calculate the optimal $A(z)$ for the initial choice of $F(z)$ ). This is repeated fixing $A(z)$ or $F(z)$ intermittently. This algorithm is guaranteed to converge, at least, to a local minimum.

In general, we have that $A_{\text {opt }}^{F(z)}(z) \neq 1$ and $F_{\text {opt }}^{A(z)}(z) \neq 0$. Thus, fixing $A(z)$ or $F(z)$ and optimally choosing the other filter, will obviously provide a coding system that enhances closed loop performance when compared with a non-coded networked situation. ${ }^{17}$ From the above, it is also clear that the use of the iterative procedure proposed above allows one to design coding systems that will always outperform simpler coding systems that are not feedback based (i.e., where $F(z)=0$; see Section 2 and also [23]). This again highlights the role of architectures (equivalently, of the available degrees of freedom) in NCSs. Further discussions regarding the interplay between coding system architecture and networked performance can be found in [23].

\subsection{Example}

Consider a nominal loop with plant and controller given by

$$
G(z)=\frac{1}{z-0.8}, \quad C(z)=\frac{z-0.8}{z-1} .
$$

The disturbance $d$ is assumed zero, whilst the reference is considered to have a power spectral density with spectral factor

$$
\Omega_{r}(z)=\frac{0.02 z}{z-0.9}
$$

We consider a finite uniform quantizer with $b$ bits and overload factor $\alpha=4$. In our design, we assume that the additive noise model for quantization holds. We send the output of the quantizer directly through the channel (i.e., without entropy coding). Thus, $b$ corresponds to the instantaneous channel data-rate in bits per sample.

Figure 8 shows the steady state tracking error variance $\sigma_{e}^{2}$ (see $(22)$ ) as a function of the number of iterations in the proposed design algorithm for $\gamma=9.1875$, which corresponds to $b=3$. Cases 1 and

\footnotetext{
${ }^{17}$ Provided both situations use the same channel and the same quantizer.
} 




Figure 8: Tracking error as function of the number of iterations in proposed design procedure (see Section 4.3 for details).



Figure 9: Tracking error as function of the channel data-rate (see Section 4.3).

2 refer to iterations that start with $A(z)=1$ and $F(z)=0$, respectively. In Case 1, we initially fixed $A(z)$, whereas in Case 2 we start by fixing $F(z)$. Case 3 refers to iterations that start with the choices suggested in [63] (where we assumed that $\gamma$ is "high enough").

In Figure 8 we have identified three points. The first of these (point (1)) refers to the performance achieved without coding $(F(z)=0$ and $A(z)=1$ ). The second (point $(2))$ refers to the performance achieved when employing the optimal coding system proposed in [23]. The third (point (3)) refers to the performance achieved using the approximately optimal filters described in [63].

The results show that coding is, indeed, necessary to achieve high closed loop performance. (Compare point (1) with, e.g., the value of $\sigma_{e}^{2}$ after 10 iterations.) It is also possible to see that use of the 
proposed procedure yields coding systems that perform better than the simpler proposals in [23, 63]; see also the discussion at the end of Section 4.2. (Compare points (2) and (3) with the limiting value for $\sigma_{e}^{2}$.)

Finally, we examine the behavior of the tracking error variance as a function of the channel datarate $b$. The results are presented in Figure 9, where "Nominal performance" refers to the performance achieved by the nominal loop (without quantization), "No coding (empirical)" refers to simulated results $^{18}$ when no coding is employed (i.e., when $A(z)=1$ and $F(z)=0$ ), "Opt. coding (empirical)" refers to simulated results obtained with the filters suggested in Section 4.2 (after 10 iterations), and "Opt. coding (analytical)" refers to the corresponding predictions made using the simplified noise model for quantization. One can see that, as expected, the effects of quantization vanish as $b \rightarrow \infty$. Interestingly, the predictions made by the additive noise model turn out to be very accurate for every bit rate: indeed, for $b \geq 3$ the relative errors are of less than $1 \%$ and, for $b \in\{1,2\}$, the relative errors are around $8 \%$. (We note that $F(z)=0$ turns out to be non-admissible for $b=1$, i.e., (23) is not satisfied. Accordingly, we have omitted the results for $b=1$ without coding.)

\section{$5 \quad$ MIMO systems}

Next we turn to MIMO systems. Our development here is largely based on [61]. We focus on control of $2 \times 2$ MIMO LTI plants models and explore the potential benefits of enhancing a traditional diagonal non-networked control architecture with additional non-transparent channels which are subject to signal-to-noise ratio constraints. We will show how to design LTI coding systems which optimize overall performance.

\subsection{Setup}

As before, $G(z)$ denotes the plant model. We assume that an admissible full MIMO controller, say

$$
C(z)=\left[\begin{array}{ll}
C_{11}(z) & C_{12}(z) \\
C_{21}(z) & C_{22}(z)
\end{array}\right]
$$

\footnotetext{
${ }^{18}$ All simulations use an actual uniform finite quantizer with $L=2^{b}$ levels. For each $b$, the results correspond to the average of 200 simulations (each one is $10^{5}$ samples long and uses a different realization for the reference signal).
} 


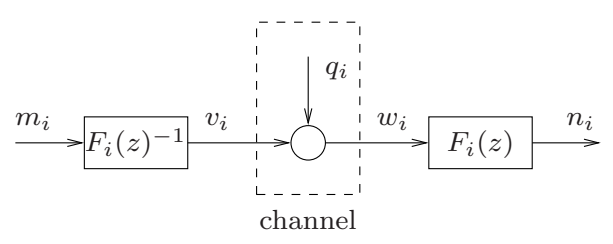

Figure 10: $i$-th communication link.

has already been designed for $G(z)$. The diagonal terms of this controller are implemented without communication constraints. However, the off-diagonal terms communicate using non transparent communication links.

We will focus on a situation where the non transparent communication links are as modeled in Figure 10. In that figure, $F_{i}(z)$ is the $i$-th $(i \in\{1,2\})$ coder transfer function, $v_{i}$ is the $i$-th channel input and $w_{i}$ is the $i$-th channel output. These signals are related via

$$
w_{i}=v_{i}+q_{i}
$$

where $q_{i}$ is the $i$-th channel noise. Each noise sequence is considered white, having variance $0 \leq \sigma_{i}^{2}<\infty$ and power spectral density $\Sigma_{i}\left(e^{j \omega}\right)=\sigma_{i}^{2}, \forall \omega \in[-\pi, \pi]$. As in previous sections, we assume that $\sigma_{i}^{2}$ is a design parameter that is proportional to the variance of the channel input (namely, proportional to $\left.\sigma_{v_{i}}^{2}\right)$. We define the associated $i$-th channel signal-to-noise ratio as

$$
\gamma_{i} \triangleq \frac{\sigma_{v_{i}}^{2}}{\sigma_{i}^{2}} .
$$

The NCS which results from employing the links described above to implement the off-diagonal terms of $C(z)$ can be visualized as in Figure 11. In that figure, $u=\left[\begin{array}{ll}u_{1} & u_{2}\end{array}\right]^{T}$ is the plant input, $y=\left[\begin{array}{ll}y_{1} & y_{2}\end{array}\right]^{T}$ is the plant output, $r=\left[\begin{array}{ll}r_{1} & r_{2}\end{array}\right]^{T}$ is the reference sequence, and $e=\left[\begin{array}{ll}e_{1} & e_{2}\end{array}\right]^{T}$ denotes the tracking error, i.e.,

$$
e \triangleq r-y
$$




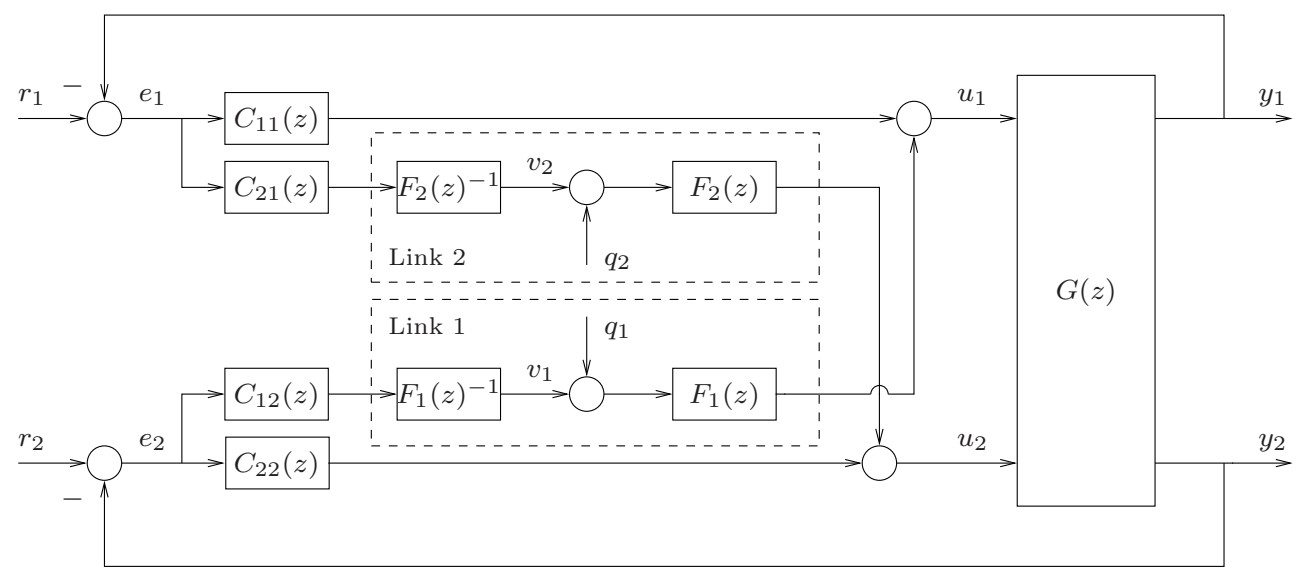

Figure 11: Partly networked MIMO control architecture (see Section 5).

\subsection{Analysis}

It is easy to see from Figure 11 that $^{19}$

$$
\begin{gathered}
\sigma_{e}^{2}=\left\|S(z) \Omega_{r}(z)\right\|_{2}^{2}+\sum_{i=1}^{2} \sigma_{i}^{2}\left\|S(z) G(z) \varepsilon_{i} F_{i}(z)\right\|_{2}^{2}, \\
\sigma_{v_{i}}^{2}=\left\|A_{i}(z) \Omega_{r}(z)\right\|_{2}^{2}+\sum_{j=1}^{2} \sigma_{j}^{2}\left\|A_{i}(z) G(z) F(z) \varepsilon_{j}\right\|_{2}^{2},
\end{gathered}
$$

where $\Omega_{r}(z)$ is a spectral factor of $r$ and

$$
\begin{aligned}
F(z) \triangleq \operatorname{diag}\left\{F_{1}(z), F_{2}(z)\right\}, & S(z) \triangleq(I+G(z) C(z))^{-1}, \\
A_{1}(z) \triangleq F_{1}(z)^{-1} C_{12}(z) \varepsilon_{2}^{T} S(z), & A_{2}(z) \triangleq F_{2}(z)^{-1} C_{21}(z) \varepsilon_{1}^{T} S(z) .
\end{aligned}
$$

Equation (28) allows one to establish that the NCS described above is MSS if and only if both $F_{1}(z)$ and $F_{2}(z)$ are stable, MP and biproper,

$$
\gamma_{1}>B_{1} \triangleq\left\|C_{12}(z) \varepsilon_{2}^{T} S(z) G(z) \varepsilon_{1}\right\|_{2}^{2}, \quad \gamma_{2}>B_{2} \triangleq\left\|C_{21}(z) \varepsilon_{2}^{T} S(z) G(z) \varepsilon_{2}\right\|_{2}^{2},
$$

and

$$
\left(\gamma_{1}-B_{1}\right)\left(\gamma_{2}-B_{2}\right)>\left\|A_{1}(z) G(z) F(z) \varepsilon_{2}\right\|_{2}^{2}\left\|A_{2}(z) G(z) F(z) \varepsilon_{1}\right\|_{2}^{2} .
$$

\footnotetext{
${ }^{19} \varepsilon_{i}$ is the $i$-th element of the canonical basis in $\mathbb{R}^{2}$.
} 
Thus, and as expected from the SISO case examined earlier, we see that MSS imposes lower bounds on the allowable channel signal-to-noise ratio.

An immediate consequence of the previous result is that, for any $C(z)$, any choice for $F_{1}(z)$ and $F_{2}(z)$, and provided (29)-(30) hold,

$$
\lim _{\left(\gamma_{1}, \gamma_{2}\right) \rightarrow\left(\bar{\gamma}_{1}, \bar{\gamma}_{2}\right)} \sigma_{e}^{2}=\infty
$$

where $\left(\bar{\gamma}_{1}, \bar{\gamma}_{2}\right)$ is any pair in

$$
\mathcal{S} \triangleq\left\{\left(\gamma_{1}, \gamma_{2}\right) \in \mathbb{R}^{2}: \gamma_{1} \text { and } \gamma_{2} \text { achieve equality in }(30)\right\}
$$

As a consequence, we see that for any given full MIMO controller, and no matter how the coding system is chosen, there exist sufficiently poor channels which render the performance of the resulting partly networked closed loop arbitrary bad. In these cases, any stabilizing decentralized controller (that makes no use of the non-transparent channels) will provide better performance than the proposed NCS. Stated a different way, for the situation under study, there are cases where sending poor information is much worse than not sending information at all. Of course, this conclusion is tied to the fact that we are considering a pre-designed controller $C(z)$. If one were to design a centralized controller considering the communication constraints from the very beginning, then one could outperform (or, at least, equal) any decentralized design.

\subsection{Coder Design}

In this section we show how to design the coders $F_{1}(z)$ and $F_{2}(z)$ under a mild simplifying assumption.

From (27) and (28) one can immediately conclude that, provided (29)-(30) are satisfied and $\gamma_{1}, \gamma_{2}$ are large enough, then $\sigma_{e}^{2} \approx J$, where

$$
J \triangleq \sum_{i=1}^{2} \frac{\left\|A_{i}(z) \Omega_{r}(z)\right\|_{2}^{2}\left\|S_{d}(z) \varepsilon_{i} F_{i}(z)\right\|_{2}^{2}}{\gamma_{i}-B_{i}} .
$$

We will denote the coders that minimize $J$ by $F_{1}^{o}(z)$ and $F_{2}^{o}(z)$.

Using the arguments of Section 2, it is possible to see that

$$
\left|F_{i}^{o}\left(e^{j \omega}\right)\right|^{4}=\beta_{i} \frac{M_{i}\left(e^{j \omega}\right) M_{i}\left(e^{j \omega}\right)^{H}}{\left(S_{d}\left(e^{j \omega}\right) \varepsilon_{i}\right)^{H} S_{d}\left(e^{j \omega}\right) \varepsilon_{i}},
$$


where

$$
M_{1}(z) \triangleq C_{12}(z) \varepsilon_{2}^{T} S(z) \Omega_{r}(z), \quad M_{2}(z) \triangleq C_{21}(z) \varepsilon_{1}^{T} S(z) \Omega_{r}(z),
$$

and $\beta_{i}$ is an arbitrary positive constant. This results shows how to synthesize coding systems that aim at minimizing the impact of the communication links on overall closed loop performance. An interesting feature of the proposed filters is that they do not depend on the channel signal-to-noise ratios $\gamma_{i}$. This allows one to conjecture that the optimal filters will perform well for a large class of communication channels.

\subsection{Example}

We end this section with an example that considers (instantaneous) bit-rate limited channels modelled using additive noise channels. For simplicity, we assume equal bit rates on each channel, i.e., $b_{1}=b_{2}=$ $b$, and take the sampling interval as $1[s]$. The plant model is given by

$$
G(z)=\left[\begin{array}{cc}
\frac{0.6}{(z-0.8)} & \frac{0.4}{(z-0.8)} \\
\frac{1}{(z-0.5)} & \frac{1}{(z-0.5)}
\end{array}\right]
$$

For this plant we (somewhat arbitrarily) synthesize the decentralized controller

$$
C_{d}(z)=\left[\begin{array}{cc}
\frac{1.3333(z-0.8)}{(z+0.8)(z-1)} & 0 \\
0 & \frac{0.8(z-0.5)}{(z+0.8)(z-1)}
\end{array}\right],
$$

and the full MIMO controller

$$
C(z)=\left[\begin{array}{cc}
\frac{5(z-0.8)}{z-1} & \frac{-2(z-0.5)}{z-1} \\
\frac{-5(z-0.8)}{z-1} & \frac{3(z-0.5)}{z-1}
\end{array}\right] .
$$

We also assume that the reference signal spectral description is given by

$$
\Omega_{r}(z)=\frac{0.0049627(z+0.9934)}{\left(z^{2}-1.97 z+0.9802\right)} I
$$

and that the quantizers are as in Section 4.3.

Figure 12 shows the tracking error variance in the proposed networked MIMO architecture as a function of the per-channel bit-rate $b$ in several situations: ${ }^{20}$ "Analytical no coding" refers to the

\footnotetext{
${ }^{20}$ The analytical results for non-integer values of $b$ correspond to cases where arbitrary values for the channel signalto-noise ratios were chosen (see (14)).
} 


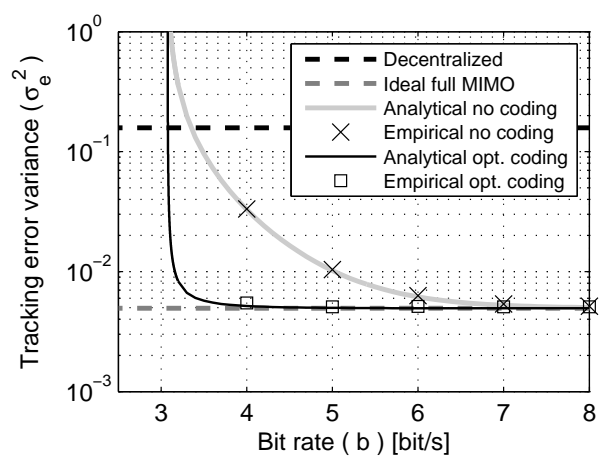

Figure 12: Tracking error variance as a function of the per-channel bit rate (see Section 5.4).

performance predicted by (27) and (28) when no coding is employed; "Empirical no coding" refers to simulated performance when no coding is considered; "Analytical opt. coding" and "Empirical opt. coding" refer to analytical and simulated performance when the coders suggested in Section 5.3 are employed. For comparison purposes, Figure 12 also shows the non networked full MIMO performance ("Ideal full MIMO") and the performance achieved when using $C_{d}(z)$ ("Decentralized"). The results allow one to conclude that, in this case, the benefits of coding are significative (compare solid-black with solid-gray lines).

As expected, non-networked full MIMO performance is recovered as $b \rightarrow \infty$, irrespective of the coders used. Moreover, it is apparent that the non coded networked full MIMO architecture should be preferred to the decentralized one for $b>3.36$. On the other hand, the optimally coded networked MIMO architecture provides significant improvement in performance for $b>3.20$, when compared to the decentralized architecture. It is also interesting to note that, if $b \rightarrow 3.07$, then the performance becomes arbitrary poor no matter what the coding is. This is consistent with (29)-(30) as straightforward calculations reveal, and brings back the question of how to actually design MIMO controllers for the considered situation (not just coding systems for a given fixed controller design).

\section{Data Loss and Delays}

Up to this point in this paper, we have focused on quantization and signal-to-noise ratio aspects in NCSs. However, there are other questions and challenges in NCSs which are more appropriately 
addressed by other paradigms. One problem that falls into the latter category is that of (non-i.i.d. ${ }^{21}$ ) data loss or delays (see, e.g., $[29,55])$. The background of this problem is that in some modern network protocols (e.g., Ethernet; see [33]), data is sent in large packets. Accordingly, quantization effects may become negligible in those situations, and the fact that data packets may get corrupted, delayed or lost, becomes the dominant issue. One strategy to deal with this problem is to encode additional information in each control packet (e.g., predicted values of future control signals). For example, in [50], the current authors present a scheme that exploits the assumption that it is possible to transmit relatively large packets that cover multiple data-dropout and delay scenarios. This provides additional information to the controller and allows remedial action to be taken in the event that packets are delayed or lost.

\section{Conclusions}

NCSs are control systems in which the communication channels between plant and controller are not transparent. Specifically, NCSs are control systems which include non standard features such as quantization (i.e. data rate limits), data dropouts and random delays. NCS theory brings together fundamental (and contemporary) ideas from control theory, information theory, signal processing and communications theory. It is, thus, an exciting area for the systems scientist to contribute to.

Significant results have been found in specific areas that allow one to analyze and synthesize networked control strategies. Nevertheless, we feel that the big picture is still missing. Many questions remain unanswered. For example, the issues of robustness to plant and channel model errors and adaptation need further work. More ambitious is the search of explicit solutions to certain kinds of problems, which would certainly increase the understanding of the interactions between control objectives and communications constraints.

Beyond technical details, the key open problem in NCSs is the development of a unified framework which takes channel issues into account and gives practice-oriented design methodologies. This makes this research area a challenging and interesting one, with a high development potential.

\footnotetext{
${ }^{21}$ Recall from Section 4 that it is possible to use the signal-to-noise paradigm to deal with i.i.d. dropouts in an exact manner [36, 62].
} 


\section{Acknowledgment}

The authors would like to thank M.S. Derpich and J. Østergaard for fundamental contributions to the work summarized here.

\section{References}

[1] P. Antsaklis and J. Baillieul. Special issue on networked control systems. IEEE Transactions on Automatic Control, 49(9):1421-1423, September 2004.

[2] P. Antsaklis and J. Baillieul. Special issue on technology of networked control systems. Proceedings of the IEEE, 95(1):5-8, January 2007.

[3] K.J. Åström. Introduction to Stochastic Control Theory. Academic Press, New York, 1970.

[4] Shun-ichi Azuma and T. Sugie. Optimal dynamic quantizers for discrete-valued input control. Automatica, 44:396-406, 2008.

[5] A. Bemporad. Predictive control of teleoperated constrained systems with unbounded communication delays. In Proc. IEEE Conf. Decis. Contr., 1998. Tampa, Florida, USA.

[6] W.R. Bennet. Spectra of quantized signals. Bell Syst. Tech. J., 27(4):446-472, 1948.

[7] H. Bölcskei and F. Hlawatsch. Noise reduction in oversampled filter banks using predictive quantization. IEEE Transactions on Information Theory, 47(1):155-172, January 2001.

[8] S. Boyd and C. Barratt. Linear Controller Design: Limits of Performance. Prentice Hall, New Jersey, 1991.

[9] S. Boyd, L. El Ghaoui, E. Feron, and V. Balakrishnan. Linear Matrix Inequalities in System and Control Theory. SIAM, 1994.

[10] J.H. Braslavsky, R.H. Middleton, and J.S. Freudenberg. Feedback stabilization over signal-to-noise ratio constrained channels. IEEE Transactions on Automatic Control, 52(8):1391-1403, 2007.

[11] C. Canudas de Wit, J. Jaglin, and K.C. Vega. Entropy coding for networked controlled systems. In Proceedings of the European Control Conference, Kos, Greece, 2007. 
[12] C. Canudas de Wit, F. Rodríguez, J. Fornés, and F. Gómez-Estern. Differential coding in networked controlled linear systems. In Proceedings of the American Control Conference, Minneapolis, USA, 2006.

[13] A. Casavola, E. Mosca, and M. Papini. Predictive teleoperation of constrained dynamic systems via internet-like channels. IEEE Trans. Contr. Syst. Technol., 14:681-694, July 2006.

[14] T.M. Cover and J.A. Thomas. Elements of Information Theory. John Wiley and Sons, Inc., 2nd edition, 2006.

[15] D.F. Delchamps. Stabilizing a linear system with quantized state feedback. IEEE Transactions on Automatic Control, 35(8):916-924, August 1990.

[16] M.S. Derpich, E.I. Silva, D.E. Quevedo, and G.C. Goodwin. On optimal perfect reconstruction feedback quantizers. IEEE Transactions on Signal Processing, 56(8):3871-3890, August 2008.

[17] N. Elia. When Bode meets Shannon: Control oriented feedback communication schemes. IEEE Transactions on Automatic Control, 49(9):1477-1488, 2004.

[18] N. Elia. Remote stabilization over fading channels. Systems \& Control Letters, 54(3):237-249, 2005.

[19] F. Fagnani and S. Zampieri. Stability analysis and synthesis for scalar linear systems with a quantized feedback. IEEE Transactions on Automatic Control, 48(9):1569-1584, September 2003.

[20] J.S. Freudenberg, R.H. Middleton, and J.H. Braslavsky. Stabilization with disturbance attenuation over a Gaussian channel. In Proceedings of the 46th IEEE Conference on Decision and Control, New Orleans, USA, 2007.

[21] J.S. Freudenberg, R.H. Middleton, and V. Solo. The minimal signal-to-noise ratio requirements to stabilize over a noisy channel. In Proceedings of the American Control Conference, Minneapolis, USA, 2006.

[22] G.C. Goodwin, S.F. Graebe, and M.E. Salgado. Control System Design. Prentice Hall, New Jersey, 2001. 
[23] G.C. Goodwin, D.E. Quevedo, and E.I. Silva. Architectures and coder design for networked control systems. Automatica, 44(1):248-257, 2008.

[24] G.C. Goodwin, M.E. Salgado, and E.I. Silva. Time-domain performance limitations arising from decentralized architectures and their relationship to the RGA. International Journal of Control, 78(13):1045-1062, September 2005.

[25] R.M. Gray and T.G. Stockham (Jr.). Dithered quantizers. IEEE Transactions on Information Theory, 39(3):805-812, May 1993.

[26] C.S. Güntürk. One-bit sigma-delta quantization with exponential accuracy. Commun. Pure Appl. Math., 56(11):1608-1630, 2003.

[27] V. Gupta, B. Sinopoli, S. Adlakha, and A. Goldsmith. Receding horizon networked control. In Proc. Allerton Conf. Communications, Control, and Computing, Monticello, IL, USA, 2006.

[28] J. Hespanha, A. Ortega, and L. Vasudevan. Towards the control of a linear system with minimum bit-rate. In Proceeding of the 15th International Symposium on Mathematical Theory of Networks and Systems (MTNS), Universty of Notre Dame, 2002.

[29] J.P. Hespanha, P. Naghshtabrizi, and Y. Xu. A survey of recent results in networked control systems. Proceedings of the IEEE, 95(1):138-162, January 2007.

[30] H. Ishii and B.A. Francis. Stabilization with control networks. Automatica, 38:1745 - 1751, 2002.

[31] N. Jayant and P. Noll. Digital Coding of Waveforms. Principles and Approaches to Speech and Video. Prentice Hall, 1984.

[32] S. Jiang and P.G. Voulgaris. Cooperative control over link limited and packet dropping networks. In Proceedings of the European Control Conference, Kos, Greece, 2007.

[33] G. Kaplan. Ethernet's winning ways. IEEE Spectrum, 38(1):113-115, 2001.

[34] V. Kariwala. Fundamental limitation on achievable decentralized performance. Automatica, 43(10):1849-1854, 2007.

[35] F. Lian, J. Moyne, and D. Tilbury. Modelling and optimal controller design of networked control systems with mutliple delays. International Journal of Control, 76(6):591-606, 2003. 
[36] Q. Ling and M.D. Lemmon. Power spectral analysis of networked control systems with data dropouts. IEEE Transactions on Automatic Control, 49(6):955-960, June 2004.

[37] G.P. Liu, J.X. Mu, D. Rees, and S.C. Chai. Design and stability analysis of networked control systems with random communication time delay using the modified MPC. International Journal of Control, 79:288-297, April 2006.

[38] I. López, C.T. Abdallah, and C. Canudas de Wit. Gain-scheduling multi-bit delta-modulator for networked controlled system. In Proceedings of the European Control Conference, Kos, Greece, 2007.

[39] N.C. Martins and M.A. Dahleh. Fundamental limitations of performance in the presence of finite capacity feedback. In Proceedings of the American Control Conference, Portland, USA, 2005.

[40] A.S. Matveev and A.V. Savkin. Decentralized stabilization of linear systems via limited capacity communication networks. In Proceedings of the 44th IEEE Conference on Decission and 2005 Control and European Control Conference, Sevilla, Spain, 2005.

[41] Y. Minami, S. Azuma, and T. Sugie. An optimal dynamic quantizer for feedback control with discrete-valued signal constraints. In Proceedings of the 46th IEEE Conference on Decision and Control, New Orleans, USA, 2007.

[42] G. Nair and R. Evans. Exponential stabilisability of finite-dimensional linear systems with limited data rates. Automatica, 39(4):585-593, April 2003.

[43] G. Nair and R. Evans. Stabilizability of stochastic linear systems with finite feedback data rates. SIAM Journal on Control and Optimization, 43(2):413-436, July 2004.

[44] G. Nair, R. Evans, I. Mareels, and W. Moran. Topological feedback entropy and nonlinear stabilization. IEEE Transactions on Automatic Control, 49(9):1585-1597, September 2004.

[45] G. Nair, F. Fagnani, S. Zampieri, and R. Evans. Feedback control under data rate constraints: An overview. Proceedings of the IEEE, 95(1):108-137, January 2007.

[46] J. Nilsson. Real-time Control Systems with Delays. PhD thesis, Lund Institute of Technology, Sweden, 1998. 
[47] D. Quevedo, J. Welsh, G. Goodwin, and M. McLeod. Networked PID control. In Proc. of the 2006 IEEE Conference on Control Applications (CCA), Munich, Germany, 2006.

[48] D.E. Quevedo, G.C. Goodwin, and J.A. De Doná. Finite constraint set receding horizon control. International Journal of Robust and Nonlinear Control, 14:355-377, March 2004.

[49] D.E. Quevedo, E.I. Silva, and G.C. Goodwin. Packetized predictive control over erasure channels. In 26th American Control Conference, New York, USA, 2007.

[50] D.E. Quevedo, E.I. Silva, and G.C. Goodwin. Control over unreliable networks affected by packet erasures and variable transmission delays. IEEE Journal on Selected Areas In Communications, 26(4):672-685, May 2008.

[51] J.B. Rawlings and B.T. Stewart. Coordinating multiple optimization based controllers: New opportunities and challenges. In Procedings of the 8th International IFAC DYCOPS, Cancún, México, 2007.

[52] A.J. Rojas, R.H. Middleton, and J.S. Freudenberg. Infimal feedback capacity for a class of additive coloured Gaussian noise channels. In Proceedings of the 17th IFAC World Congress, Seoul, Korea, 2008.

[53] M.E. Salgado and A. Conley. MIMO interaction measure and controller structure selection. International Journal of Control, 77(4):367-383, March 2004.

[54] A.V. Savkin. Analysis and synthesis of networked control systems: Topological entropy, observability, robustness and optimal control. Automatica, 42:51-62, 2006.

[55] L. Schenato, B. Sinopoli, M. Franceschetti, K. Poolla, and S.S. Sastry. Foundations of control and estimation over lossy networks. Proceedings of the IEEE, 95(1):163 - 187, January 2007.

[56] R. Schreier and G.C. Temes. Understanding Delta Sigma Data Converters. Wiley-IEEE Press, 2004.

[57] P. Seiler and R. Sengupta. An $\mathcal{H}_{\infty}$ approach to networked control. IEEE Transactions on Automatic Control, 50(3), 2005. 
[58] C.E. Shannon. A mathematical theory of communication. The Bell System Technical Journal, 27:379-423, 623-656, July, October 1948.

[59] E.I. Silva. A Unified Framework for the Analysis and Design of Networked Control Systems. PhD thesis, School of Electrical Engineering and Computer Science, The University of Newcastle, Australia, 2009.

[60] E.I. Silva, M.S. Derpich, J. Østergaard, and D.E. Quevedo. Simple coding for achieving mean square stability over bit-rate limited channels. In Proceedings of the 46 th IEEE Conference on Decision and Control, Cancún, México, 2008.

[61] E.I. Silva, G.C. Goodwin, and D.E. Quevedo. On networked control architectures for MIMO plants. In Proceedings of the 17th IFAC World Congress, Seoul, Korea, 2008.

[62] E.I. Silva, G.C. Goodwin, and D.E. Quevedo. On the design of control systems over unreliable channels. Budapest, Hungary, Submitted to the European Control Conference 2009.

[63] E.I. Silva, G.C. Goodwin, D.E. Quevedo, and M.S. Derpich. Optimal noise shaping for networked control systems. In Proceedings of the European Control Conference, Kos, Greece, 2007.

[64] E.I. Silva, D.A. Oyarzun, and M.E. Salgado. On structurally constrained $\mathcal{H}_{2}$ performance bounds for stable MIMO plant models. IET Control Theory \& Applications, 1(4):1033-1045, 2007.

[65] E.I. Silva, D.E. Quevedo, M.S. Derpich, and G.C. Goodwin. Design of feedback quantizers for networked control systems. Technical report, School of Electrical Engineering and Computer Science, The University of Newcastle, Australia, 2008.

[66] E.I. Silva, D.E. Quevedo, and G.C. Goodwin. Optimal controller design for networked control systems. In Proceedings of the 17th IFAC World Congress, Seoul, Korea, 2008.

[67] S. Skogestad and I. Postlethwaite. Multivariable Feedback Control: Analysis and Design. Wiley, New York, 1996.

[68] P.L. Tang and C.W. de Silva. Compensation for transmission delays in an ethernet-based control network using variable-horizon predictive control. IEEE Transactions on Control and Systems Technology, 14(4):707-718, July 2006. 
[69] S. Tatikonda and S. Mitter. Control over noisy channels. IEEE Transactions on Automatic Control, 49(7):1196-1201, July 2004.

[70] S. Tatikonda and S. Mitter. Control under communication constraints. IEEE Transactions on Automatic Control, 49(7):1056-1068, July 2004.

[71] S. Yüksel and T. Başar. Communication constraints for decentralized stabilizability with timeinvariant policies. IEEE Transactions on Automatic Control, 52(6):1060-1066, 2007.

[72] R. Zamir and M. Feder. On universal quantization by randomized uniform/lattice quantizers. IEEE Transactions on Information Theory, 38(2):428-436, March 1992.

[73] R. Zamir and M. Feder. Information rates of pre/post-filtered dithered quantizers. IEEE Transactions on Information Theory, 42(5):1340-1353, September 1996.

[74] K. Zhou, J.C. Doyle, and K. Glover. Robust and optimal control. Prentice Hall, Englewood Cliffs, N.J., 1996. 